\title{
Cardiac tamponade due to group a streptococcal pericarditis in a 10-month-old boy and a review of the literature
}

\author{
Matthew C. Schwartz ${ }^{1 *}$, Matthew J. Gillespie ${ }^{1}$, Paul Stephens Jr. ${ }^{1}$, Brian Fisher ${ }^{2}$ \\ ${ }^{1}$ Division of Cardiology, The Children's Hospital of Philadelphia, Philadelphia, USA; \\ ${ }^{2}$ Division of Infectious Disease and Center for Clinical Epidemiology and Biostatistics, The Children's Hospital of Philadelphia, \\ Philadelphia, USA. \\ Email: schwartzm@email.chop.edu
}

Received 18 September 2011; revised 27 October 2011; accepted 8 November 2011.

\begin{abstract}
Group A streptococcus (GAS) is a rare cause of purulent pericarditis in pediatric patients as only 7 cases have been reported. We present a 10-month-old boy who developed cardiac tamponade from GAS and was successfully treated with subxiphoid tube drainage and 4 weeks of antibiotics at the Children's Hospital of Philadelphia.
\end{abstract}

Keywords: Streptococcus; Pericarditis; Pericardiocentesis; Tamponade

\section{INTRODUCTION}

Group A streptococcus (GAS) is a rare cause of bacterial pericarditis in children with only 7 pediatric cases previously reported [1-5]. We describe a 10-month-old who developed cardiac tamponade due to GAS pericarditis and was successfully treated.

\section{CASE REPORT}

A 10-month-old boy presented to the Children's Hospital of Philadelphia (CHOP) Emergency Department (ED) with 9 days of fever accompanied by a transient papular rash, but no other localizing symptoms. He underwent outpatient evaluation on day 6 and was diagnosed with roseola. The patient's fever continued prompting his presentation.

His birth and past medical histories were unremarkable. He lived in Philadelphia and had no exposures or travel. Several days into the child's illness, his father was diagnosed with GAS pharyngitis by rapid streptococcal antigen test and was treated.

In the ED, he had a rectal temperature of $38.2^{\circ} \mathrm{C}$. The remainder of his vital signs and physical exam were normal except for mild bilateral anterior cervical lymphadenopathy. Initial laboratory testing showed a white blood cell count of 20,500/uL (63\% neutrophils, 26\% lymphocytes), a hemoglobin of $10.2 \mathrm{~g} / \mathrm{dL}$, a platelet count of 57,400/uL, an erythrocyte sedimentation rate of $75 \mathrm{~mm} / \mathrm{hr}$, a C-reactive protein of $6.2 \mathrm{mg} / \mathrm{dL}$, an albumin of $3.7 \mathrm{~g} / \mathrm{dL}$, an aspartate aminotransferase of $109 \mathrm{U} / \mathrm{L}$, and an alanine aminotransferase $<6 \mathrm{U} / \mathrm{L}$. Urinalysis and chest radiograph were normal. Urine and blood cultures were sent as was a respiratory virus polymerase chain reaction (PCR) panel on respiratory secretions.

The patient was admitted for evaluation. On hospital day 1 , the respiratory virus PCR panel returned negative for adenovirus, respiratory syncytial virus, influenza $A$ and $\mathrm{B}$, parainfluenza 1 - 3, metapneumovirus, and rhinovirus. Serum was sent for adenovirus PCR, human herpes virus-6 (HHV6) PCR, cytomegalovirus (CMV) PCR, and Ebstein Barr virus (EBV) serology. To consider occult osteomyelitis, a whole body magnetic resonance imaging scan was obtained and was negative. In light of possible Kawasaki Disease, an echocardiogram was performed, revealing a small, $4 \mathrm{~mm}$ posterior pericardial effusion, normal biventricular systolic function, and mild, diffuse ectasia of the right coronary artery (proximal right coronary artery z-score 2.62).

Due to fever, elevated inflammatory markers, an elevated aspartate transferase, and ectasia of the right coronary artery, incomplete Kawasaki Disease was suspected. On hospital day 2, the patient was treated with intravenous immunoglobulin and aspirin was started. From hospital day 3 through 5 , the patient's fever continued and his inflammatory markers increased. Serial blood cultures remained negative and urine culture at the time of admission was negative. The serum adenovirus PCR, CMV PCR, and EBV serologies returned negative. The patient's HHV6 serum PCR returned as mildly positive (1981 copies/mL). The Infectious Disease Service was consulted and felt HHV6 not likely responsible for the illness.

By hospital day 6, the patient remained febrile and had increased work of breathing. Chest x-ray showed an 
enlarged cardiac silhouette and echocardiogram showed a large circumferential pericardial effusion $(1.2 \mathrm{~cm})$ with early signs of tamponade physiology including right atrial collapse in late ventricular diastole (Figure 1). The coronary arteries appeared normal with no evidence of right coronary artery ectasia. An electrocardiogram showed sinus tachycardia with no ST segment or PR segment changes.

The patient was transferred to the Intensive Care Unit and pericardiocentesis drained 65 cc of purulent fluid and a drain left in place. The gram stain showed gram positive cocci and empiric antibiotics were started. The bacteria was subsequently identified as Group A beta hemolytic streptococcus and the antibiotics changed to intravenous penicillin $\mathrm{G}$. The patient improved and the pericardial drain was removed several days after pericardiocentesis. The patient completed 14 days of intravenous antimicrobials following drainage and was discharged home to complete another 14 days of oral penicillin (total 4 week course).

Four months after discharge, the patient was well and echocardiogram showed a normal pericardial sac.

\section{DISCUSSION}

Pericarditis is uncommon in children. Of 20 cases reported by Roodpeyma et al., 8 (40\%) were bacterial, 6 (30\%) associated with collagen vascular disease, 4 (20\%) were viral, and $2(10 \%)$ due to mass invasion of the pericardium [6]. Pericarditis can also be related to postpericardiotomy syndrome or can be idiopathic.

Clinical signs of purulent pericarditis include fever, tachycardia, tachypnea, hepatomegaly, and decreased

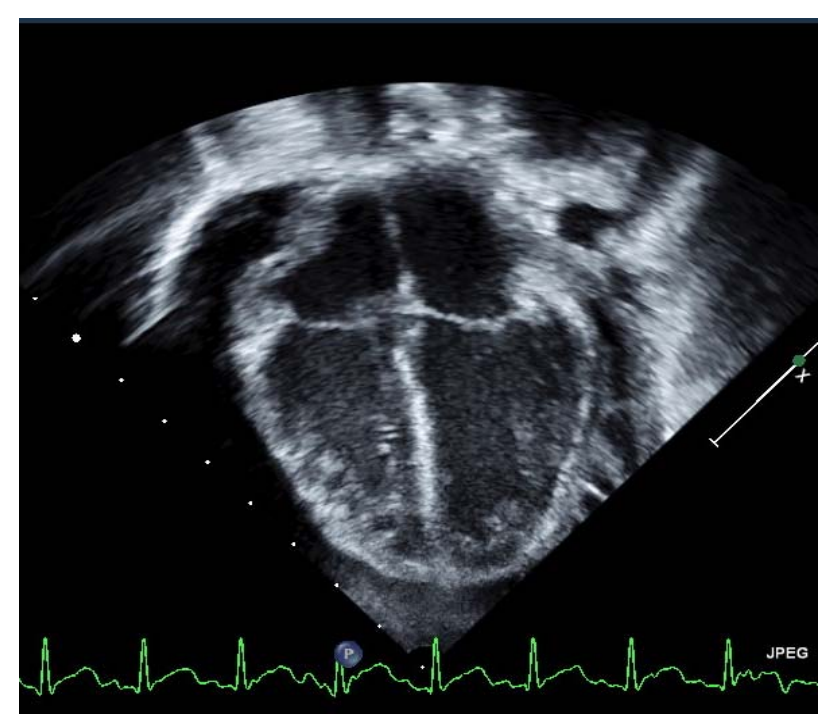

Figure 1. Transthoracic apical four chamber view showing a large, circumferential pericardial effusion with fibrinous strands and diastolic right atrial collapse consistent with tamponade physiology. cardiac sounds. Patients rarely present with pericardial friction rub because the pericardial space is distended with pus. Electrocardiogram often shows decreased voltages and diffuse ST elevation and chest x-ray usually shows an enlarged cardiac shadow. All patients will have significant pericardial fluid by echocardiography and many children with bacterial pericarditis will present with cardiac tamponade [2,7].

Our patient presented in a more indolent fashion with long-standing fever. It is possible that the illness was two separate infections such as a viral syndrome followed by invasive GAS. GAS invasive disease occurring in the setting of viral infection has been described although association of GAS infection with HHV-6 has not been reported $[8,9]$. The patient had a mildly positive serum HHV-6 PCR on day 9 of his illness which raises the possibility of an initial HHV-6 infection, but is not conclusive.

Purulent pericarditis commonly develops secondary to a primary bacterial infection at an alternate location, most commonly pneumonia [2,7]. Septic emboli or direct spread from the lungs then acts to seed the pericardium. In our patient, the pericardium likely was the primary site of invasive infection.

The most common cause of bacterial pericarditis in children is Staphylococcus aureus [6,7]. Streptococcus pneumoniae and Haemophilus influenzae are also sometimes isolated [2,7]. GAS, however, is a rare cause. Review of the literature shows that only 7 cases have ever been reported in pediatric patients (Table 1) [1-5]. One case was not described in detail [2], but the ages of the other 6 cases range from 13 months to 14 years with 3 males and 3 females. Our patient is the youngest patient reported with this entity. Three patients had an identifiable source of GAS infection; 1 patient had pharyngitis and cellulitis, 1 patient pharyngitis alone, and another patient had pneumonia. All were treated with penicillin or a penicillin derivative except one, whose antibiotic regimen was not described. In the 2 reports that described the entire course of antibiotics, both patients received four weeks of therapy [1,5]. All underwent pericardiocentesis with isolation of GAS from pericardial fluid. Two patients also underwent surgical pericardial intervention. One of the 6 patients died; a 2 year old female was diagnosed with viral pharyngitis several days before presenting in respiratory distress and deteriorating shortly after admission despite pericardiocentesis [3]. Of the 5 surviving patients, none developed constrictive pericardial disease with limited follow-up.

Systemic antibiotics and pericardial drainage are both needed to treat of purulent pericarditis. Antibiotics must include an antistaphylococcal agent until the organism is identified. There are no guidelines regarding length of 
Table 1. Previously reported cases of purulent pericarditis in children due to Group A Streptococcus.

\begin{tabular}{|c|c|c|c|c|c|c|c|}
\hline Reference & Year Treated & Age & Sex & Antibiotic & Source & Drainage & Survival \\
\hline \multicolumn{8}{|l|}{ Gersony, et al. $1967^{*}$} \\
\hline Vigneswaran, et al. 1985. & Not documented & 14 yo & M & $\begin{array}{l}\text { Penicillin } \mathrm{G} \times 10 \text { days, } \\
\text { then oral Penicillin } \\
\mathrm{V} \times 4 \text { weeks }\end{array}$ & $\begin{array}{c}\text { Pharyngitis, } \\
\text { Cellulitis }\end{array}$ & $\begin{array}{l}\text { Pericardiocentesis, } \\
\text { Pericardial window }\end{array}$ & Alive, f/u 8 weeks \\
\hline Thebaud, et al. 1996. & 1979 & $13 \mathrm{mo}$ & M & $\begin{array}{l}\text { Ampicillin, } \\
\text { Gentamicin }\end{array}$ & $\begin{array}{l}\text { No extracardiac } \\
\text { source }\end{array}$ & Pericardiocentesis & Not documented \\
\hline Thebaud, et al. 1996. & 1981 & 3 yo & $\mathrm{F}$ & $\begin{array}{l}\text { Oxacillin, } \\
\text { Gentamicin }\end{array}$ & $\begin{array}{c}\text { No extracardiac } \\
\text { source }\end{array}$ & Pericardiocentesis & Not documented \\
\hline Thebaud, et al. 1996. & 1985 & $14 \mathrm{mo}$ & $\mathrm{F}$ & $\begin{array}{l}\text { Amoxicillin, } \\
\text { Neomycin }\end{array}$ & Pneumonia & Pericardiocentesis & Not documented \\
\hline Pruitt, et al. 1989. & 1987 & 2 yo & $\mathrm{F}$ & $\begin{array}{c}\text { Not } \\
\text { documented }\end{array}$ & Pharyngitis & Pericardiocentesis & $\begin{array}{l}\text { Died 4d after } \\
\text { presentation }\end{array}$ \\
\hline
\end{tabular}

${ }^{*}$ Review does not include details of case.

antimicrobial therapy, but most patients are treated with 2 to 4 weeks of intravenous therapy [1,5,7]. Several options for pericardial drainage exist including subxiphoid tube placement, pericardial window creation, partial pericardiectomy, total pericardiectomy, and/or infusion of thrombolytics into the pericardial space following pericardiocentesis. Factors such as the consistency of the fluid, the patient's clinical status, and the cause of pericarditis influence which modality is chosen.

Even with therapy, the mortality in bacterial pericarditis in children ranges from $5 \%$ to $12 \%[1,6,7]$. Our patient was successfully treated with antibiotics and subxiphoid tube drainage without evidence of pericardial constriction 4 months after treatment completion.

\section{REFERENCES}

[1] Bhaduri-McIntosh, S., Prasad, M., Moltedo, J. and Vazquez, M. (2006) Purulent pericarditis caused by group A streptococcus. Texas Heart Institute Journal, 33, 519-522.

[2] Gersony, W.M. and McCracken, G.H. (1967) Purulent pericarditis in infancy. Pediatrics, 40, 224-232.

[3] Pruit, J.L. (1989) Group A streptococcal pericarditis in a previously well child. Pediatric Infectious Disease Journal, 8, 338.

[4] Thebaud, B., Sidi, D. and Kachaner, J. (1996) Les pericardites purulentes de l'enfant: 15 ans d'experience. Archives of Pediatrics, 3, 1084-1090. doi:10.1016/S0929-693X(96)89513-3

[5] Vigneswaran, W.T., Hardie, R., Ferguson, J.C. and Faichney, A. (1985) Cardiac tamponade due to lancefield group A beta haemolytic streptococcal pericarditis. Thorax, 40, 549-555. doi:10.1136/thx.40.7.549

[6] Roodpeyma, S. and Sadeghian, N. (2000) Acute pericarditis in childhood: A 10-year experience. Pediatric Cardiology, 21, 363-367. doi:10.1007/s002460010081

[7] Cakir, O., Gurkan, F., Balci, A.E., Eren, N. and Dikici, B. (2002) Purulent pericarditis in childhood: Ten years of experience. Journal of Pediatric Surgery, 37, 1404-1408. doi:10.1053/jpsu.2002.35401

[8] Harre, B. (2006) Fatal group A streptococcal myopericarditis during influenza A infection. Pediatric Infectious Disease Journal, 25, 660-661.

doi:10.1097/01.inf.0000224544.05879.62

[9] Sivakumar, S. and Latifi, S.Q. (2008) Varicella with stridor: Think group A streptococcal epiglottitis. Journal of Paediatrics and Child Health, 44, 149-151. doi:10.1111/j.1440-1754.2007.01280.x 